\title{
Immunologic impact of the intestine in metabolic disease
}

\author{
Daniel A. Winer, ${ }^{1,2,3,4}$ Shawn Winer, ${ }^{3,5}$ Helen J. Dranse, ${ }^{6,7}$ and Tony K.T. Lam ${ }^{4,6,7}$ \\ ${ }^{1}$ Department of Pathology and Toronto General Hospital Research Institute, UHN, Toronto, Ontario, Canada. ${ }^{2}$ Department of Immunology, ${ }^{3}$ Department Laboratory Medicine and Pathobiology, and ${ }^{4}$ Banting \\ and Best Diabetes Centre, University of Toronto, Toronto, Ontario, Canada. ${ }^{5}$ Department of Laboratory Medicine, St. Michael's Hospital, Toronto, Ontario, Canada. ${ }^{6}$ Toronto Ceneral Hospital Research Institute \\ \& Department of Medicine, UHN, Toronto, Ontario, Canada. Departments of Physiology and Medicine, University of Toronto, Toronto, Ontario, Canada.
}

\begin{abstract}
Obesity and diabetes are associated with increased chronic low-grade inflammation and elevated plasma glucose levels. Although inflammation in the fat and liver are established features of obesity-associated insulin resistance, the intestine is emerging as a new site for immunologic changes that affect whole-body metabolism. Specifically, microbial and dietary factors incurred by diet-induced obesity influence underlying innate and adaptive responses of the intestinal immune system. These responses affect the maintenance of the intestinal barrier, systemic inflammation, and glucose metabolism. In this Review we propose that an understanding of the changes to the intestinal immune system, and how these changes influence systemic immunity and glucose metabolism in a whole-body integrative and a neuronal-dependent network, will unveil novel intestinal pathologic and therapeutic targets for diabetes and obesity.
\end{abstract}

\section{Introduction}

Obesity-associated insulin resistance is a precursor to type 2 diabetes and is part of the metabolic syndrome, which has reached epidemic proportions worldwide. Multiple factors contribute to insulin resistance and glucose dysregulation in obesity and diabetes, but chronic low-grade inflammation of metabolic tissues, including visceral adipose tissue (VAT), liver, and brain, are important contributors (1-11). The intestinal immune system has recently emerged as a site that is altered by diet-induced obesity. The specific influence of the gut microbiota on the development of metabolic disorders, and how bacterial signals compartmentalized in the gut directly shape immune responses linked to systemic and metabolic tissue inflammation, are active areas of interest and have been previously reviewed (12-14). In addition to microbial signals, dietary cues also help shape the composition of the intestinal immune system, and these effects collectively contribute to its role as a novel regulator of metabolic disease. In this Review we aim to highlight some of the intestinal immunologic events that are altered in nutrient excess or obese conditions in response to both microbial and dietary factors, and pinpoint how such changes may influence systemic immunologic and glucose homeostasis via an integrative and neuronal-dependent network.

\section{The intestinal immune system}

The intestinal immune system maintains immunologic tolerance to commensal bacterial and dietary antigens. This maintenance is governed by innate and adaptive immune systems located along the intestinal epithelial surface and lamina propria that form the intestinal barrier. Mucus, antimicrobial peptides (AMPs), intestinal epithelial cells (IECs), microfold cells, paneth cells, innate lymphoid

Conflict of interest: The authors have declared that no conflict of interest exists Reference information: / Clin Invest. 2017;127(1):33-42. doi:10.1172/JCI88879. cells (ILCs), and other rapid-response immune cells make up the innate system. The adaptive immune system is composed of $\mathrm{T}$ cells, B cells, and plasma cells with their secreted antibodies, including IgA. B and T cells are frequently arranged within the gut-associated lymphoid tissue (GALT), including Peyer's patches (distal ileum), isolated lymphoid follicles, and mesenteric lymph nodes. The intestinal immune system function is dictated by responses elicited by sensing of microbe-associated molecular patterns (MAMPs) expressed by commensal gut flora or pathogens. These MAMPs are detected via pattern-recognition receptors (PRRs) such as the TLRs, nuclear oligomerization domain-like receptors (NLRs), and RIG-I like receptor in IECs and immune cells (15).

To maintain eubiotic homeostasis, goblet cells secrete mucus composed of glycosylated mucins and protective molecules like trefoil factor $(16,17)$, while IECs and Paneth cells produce AMPs and C-type lectin regenerating islet-derived protein III $\gamma(\operatorname{RegIII} \gamma)$ to exclude bacteria (18). In addition, IECs secrete thymic stromal lymphopoietin (TSLP), TGF- $\beta$, retinoic acid, and IL- 25 to promote immune tolerance and barrier function (19). The immune system switches to an inflammatory state upon pathogen invasion or barrier breech. MAMPs stimulate the secretion of the proinflammatory cytokines IL-1, IL-6, IL-12, IL-18, and/or IL-23 from IECs as well as underlying DCs and macrophages (20).

\section{Microbial and dietary influences on intestinal immunity}

There are a number of microbial and dietary influences on the intestinal immune system that occur with obesity. These changes and consequent influences on intestinal immunity and barrier function are described below and are collectively summarized in Figure 1.

Microbiota. The development and function of the intestinal immune system is influenced by the gut microbiota, including bacteria with the capacity to induce specific lineage differentiation in immune cells. Germ-free mice have an underdeveloped intestinal 


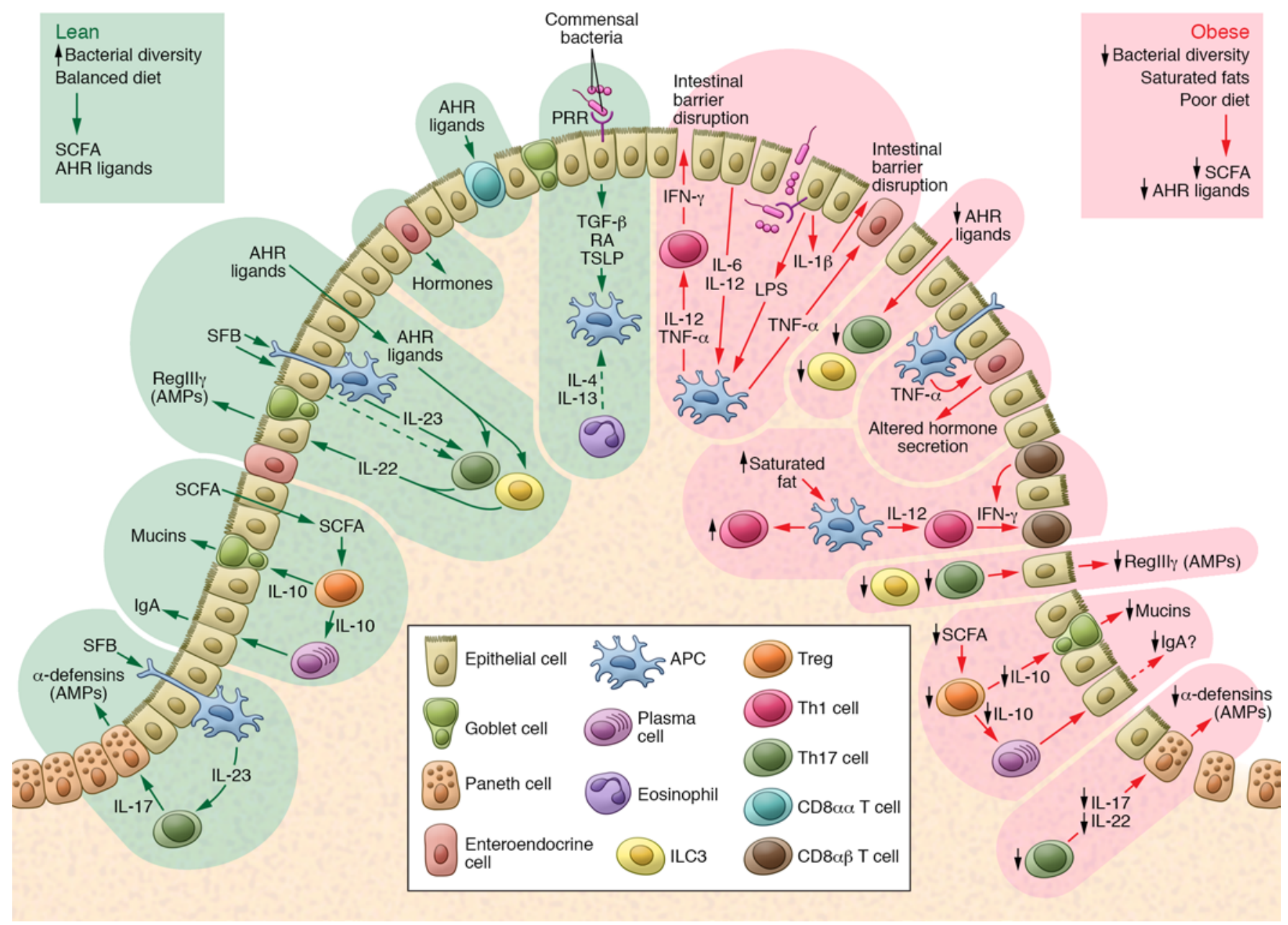

Figure 1. Dietary and microbial cues influence intestinal immunity during diet-induced obesity. During intestinal homeostasis in the lean state (left), microbial metabolites, including SCFAs (e.g., butyrate), improve intestinal barrier function through promotion of Treg function. Treg-derived IL-10 in turn may boost mucin production. Apical signaling of commensal organisms through select PRRs also promote production of the largely antiinflammatory mediators TGF- $\beta$, retinoic acid (RA), and TSLP, which maintains intestinal tolerance. Products such as AHR ligands derived from dietary fruits and vegetables fuel the function of groups of immune cells (e.g., ILC3s and CD8 $\alpha \alpha$ T cells; arrows lead from AHR ligands to ILC3s and Th17 cells), which produce mediators that maintain the epithelial barrier. During diet-induced obesity (right) there is an increase in saturated fats coupled with a reduction in fibers and associated bacterial dysbiosis. These alterations lead to a state of low-grade chronic inflammatory changes in local immune populations, which have numerous effects on intestinal homeostasis. The relative reductions in IL-10, coupled with increasing local TNF- $\alpha$, may compromise mucin production and IgA class switching and affect gut hormone secretion. The restriction in bacterial diversity, coupled with reductions in AHR ligands from a lack of fruits and vegetables, leads to a reduction in ILC3s, CD8 $\alpha \alpha$ T cells, and Th17 cells, which maintain barrier integrity. This overall reduction in intestinal barrier integrity promotes leakage of bacterial products such as LPS, which helps support a shift in T cell polarity to IFN- $\gamma$-releasing Th1 T cells. The ensuing low-grade inflammation further compromises intestinal barrier function, resulting in the characteristic permeable barrier associated with diet-induced obesity.

immune system, signifying a role for MAMPs in its establishment (21). Some commensal bacteria such as segmented filamentous bacteria (SFBs) preferentially induce a Th17 response in T cells $(22,23)$. Other commensals or their products guide an IL-10 Treg response. The capsular polysaccharide-A moiety on Bacteroides fragilis promotes expansion of Foxp $3^{+}$Tregs through a TLR2/MyD88 mechanism (24), while Clostridium species can induce Treg formation independent of MyD88, potentially through TGF- $\beta$ (25). Bacterial metabolites like short-chain fatty acids (SCFA) produced by Akkermansia muciniphila and Faecalibacterium prausnitzii also influence immunity. For instance, butyrate from these organisms increases the differentiation of Tregs in the colon $(26,27)$.

In obesity, there is a change in the gut microbial content known as dysbiosis. Most studies show that dysbiosis is associated with an increase in the ratio of Firmicutes to Bacteroidetes phyla in the gut, changes that have been associated with increased energy harvesting from food and increased triglyceride storage in liver and fat $(28,29)$. Dysbiosis imparts features of metabolic syndrome. For instance, the obese phenotype can be transferred from obese humans to mice through transplant of gut microbiota (30). Recently, human metagenomics studies have linked gut microbial dysbiosis to features of metabolic syndrome (31-33). For example, low bacterial gene count in fecal DNA (low bacterial richness) correlates with dyslipidemia, insulin resistance, and inflammation, whereas high gene count is associated with antiinflammatory species including $F$. prausnitzii and production of SCFAs such as butyrate (32). Another study showed that patients with untreated type 2 diabetes exhibit a reduction in butyrate-producing bacte- 
ria, and that this reduction is reversed with metformin treatment (34). This study indicates that in addition to dietary factors, there are also pharmacologic confounders, including metformin, which may also exert selective pressures to shape the microbiota.

An important effect of high-fat diet-associated (HFDassociated) dysbiosis is the change to intestinal epithelial barrier integrity and intestinal permeability. A compromised epithelial barrier allows for translocation of food antigen and bacterial products from the gut to the tissues, including metabolic tissues like liver, adipose, and brain, which may potentiate local immune cell stimulation. Transepithelial transport of bacterial components such as LPS are thought to occur through two primary mechanisms, paracellular and transcellular transport, both of which are increased in obesity. For instance, HFD-fed mice have decreased levels of tight junction proteins, resulting in increased intestinal permeability (35). Alternatively, transcytosis, whereby LPS is transported into enterocytes, incorporated into lipid-rich chylomicrons, and secreted in transport vesicles into circulation, is increased following a HFD as a consequence of increased chylomicron formation and release (36). It may also be possible that bacterial products can enter the intestinal barrier through goblet cell endocytosis (37), though further work is needed to determine whether this process is involved mechanistically in the setting of bacterial transcellular permeability during HFD feeding. Leakage of bacterial LPS across the barrier induces low-grade inflammation and HFD-associated metabolic abnormalities in a process termed "metabolic endotoxemia" $(35,38,39)$. Recent studies have linked dysbiosis associated with a lard-based HFD to metabolic inflammation, whereby leaked gut microbial products stimulate adipocyte TLR4, MyD88, and TLR adaptor molecule 1 (TRIF) signaling, to facilitate CCL2-mediated immune cell inflammation in adipose tissue (40).

Dietary factors. The intestinal immune system is also strongly shaped by dietary factors. Fruits, nuts, and vegetables are sources of ligands for the aryl hydrocarbon receptor (AHR) transcription factor. Mice lacking AHR or fruits and vegetables in their diet show reduced intestinal lymphoid tissue development and reduced numbers and function of intraepithelial lymphocytes (including CD $8 \alpha \alpha$ $\mathrm{T}$ cells and $\gamma \delta \mathrm{T}$ cells) and ROR $\gamma \mathrm{t}^{+}$ILCs (41). AHR function is also important for ILC-derived IL-22 responses, which promote intestinal barrier function (42). The lack of such protective effects on the intestinal barrier may be exacerbated in obesity and by a Western diet, where the effects of reduced fruits and vegetables likely synergize with dysbiosis to promote weakened barrier integrity.

The digestion of dietary fats gives rise to fatty acids. Plantand fish-based diets typically yield polyunsaturated fatty acids, while a Western diet rich in red meat and fried foods is a source of saturated fatty acids. In general, saturated fatty acids induce inflammatory activation of immune cells, while polyunsaturated fats can give rise to both inflammatory and antiinflammatory functions (43). For instance, saturated fatty acids activate TLR2 and TLR4 signaling in macrophages to promote inflammatory cytokine release (44), while the omega-3 polyunsaturated fatty acids bind the GPCR GPR120 to reduce inflammatory activity (45). Cholesterol metabolites such as oxysterols or bile acids can activate nuclear receptors such as the liver X receptor (LXR) and farnesoid $\mathrm{X}$ receptor (FXR) to modulate intestinal immunity.
The LXR reduces Th17 lineage differentiation (46), as this effect competes with endogenous oxysterol ligands for ROR $\gamma \mathrm{t}$ (47) while gut-specific targeting of the FXR exerts antiinflammatory effects (48). A fat-enriched diet also reduces the number of ileal IL-17/ROR $\gamma \mathrm{t} \mathrm{CD} 4^{+} \mathrm{T}$ cells, and IL-17/ROR $\gamma \mathrm{t}-$ deficient T cells are associated with the induction of glucose intolerance and obesity (49). Metabolites of vitamins A and D bind nuclear hormone receptors such as the retinoic acid receptor and the vitamin D receptor, which can dimerize with retinoid $\mathrm{X}$ receptors to alter transcriptional programs in innate and adaptive immune cells. These vitamins show broad effects on immunologic tolerance and function $(50,51)$; however, their roles in regulating metabolic disease require further investigation.

Regulators of intestinal barrier function in diet-induced obesity. The means by which dysbiosis and diet-induced obesity alter epithelial integrity is an area of active investigation. Gut bacterial signals are critical in regulating key barrier components such as mucin, AMPs, and secretory IgA. During dysbiosis, there is a selection for distinct types of bacteria, which may alter metabolite production as well as barrier and immune function (52). An example is the aforementioned reduction in SCFA butyrate-producing bacteria in patients with type 2 diabetes (32). SCFA is implicated in epithelial barrier maintenance by promoting mucus expression by IECs, IgA expression in B cells, tissue repair, Treg differentiation, IL-18-related epithelial integrity, and inhibition of NF- $\mathrm{\kappa B}(53,54)$. Another bacterial metabolite, indole-3-aldehyde, is generated through metabolism of tryptophan in some microbiota (55). In this case, bacterial tryptophan metabolites serve as ligands for the AHR in intestinal immune cells. AHR activity is directly involved in production of cytokines critical in maintaining barrier function, including IL-22 by ROR $\gamma$ t ILCs and Th17 cells $(42,56)$. Thus, when coupling dysbiosis with a diet lacking fruits and vegetables, which are a rich source of such AHR ligands, there is a compounded effect on immunologic maintenance of barrier integrity.

Changes in intestinal AMPs and cytokines downstream of dietary and microbial stimuli represent an important immunologic influence on gut barrier integrity during obesity. For example, during HFD-induced obesity in mice, a reduction in production of AMPs RegIII $\beta$ and RegIII $\gamma$ is identified as early as ten days after starting a HFD; these changes in AMPs contribute to worsening permeability (49). Cytokines such as IL-1 $\beta$ and IFN- $\gamma$, which are increased in the gut during HFD feeding, can worsen intestinal barrier integrity through direct action on the expression of epithelial tight junction proteins such as occludin and zonula occludens $1(57,58)$. Such cytokines are countered by ones that can promote barrier integrity, such as IL-22, IL-25, IL-17, and IL-10. IL-10 in particular maintains the barrier through promotion of mucin production by goblet cells (59). Inflammatory effects on mucosal barrier function may be further influenced by genetic factors such as polymorphisms in immunologic loci, including TLR4 or IL-10 (60-62). Future areas of investigation include understanding the triggers for inflammatory cytokine production during diet-induced obesity and understanding how the fundamental cellular processes perturbed during obesity, such as autophagy and ER stress, impinge on cytokine release and intestinal barrier integrity in obesity $(63,64)$. 
Table 1. Summary of changes to intestinal immune cell subsets in the obese state compared with the lean state

\begin{tabular}{|c|c|c|c|}
\hline \multicolumn{3}{|l|}{ Innate changes } & Citation \\
\hline ILC3 (NCR+CD4 $\left.{ }^{-}\right)$ & NA & $\downarrow$ (mouse) & 58 \\
\hline \multirow[t]{2}{*}{$\gamma \delta \mathrm{T}$ cell } & $\uparrow$ (mouse) & $\uparrow$ (mouse) & 58 \\
\hline & $\leftrightarrow$ (human) & NA & 77 \\
\hline MAIT cell & NA & NA & 79 \\
\hline Eosinophil & $\downarrow$ (mouse) & NA & 80 \\
\hline \multirow[t]{2}{*}{ Macrophage } & $\uparrow$ (human) & NA & 77 \\
\hline & $\leftrightarrow$ (mouse) & NA & 49 \\
\hline \multirow[t]{2}{*}{$D C$} & $\uparrow$ (human, diabetes) & NA & 77 \\
\hline & $\leftrightarrow$ (mouse) & NA & 49 \\
\hline NK cell & $\uparrow$ (human, diabetes) & NA & 77 \\
\hline Mast cell & $\leftrightarrow$ (human) & NA & 77 \\
\hline \multicolumn{4}{|l|}{ Adaptive changes } \\
\hline \multirow[t]{2}{*}{ CD8 ${ }^{+} \mathrm{T}$ cell ( $\alpha \beta$ TCR) } & $\uparrow$ (mouse, human) & $\uparrow$ (mouse, human) & 58 \\
\hline & $\uparrow$ (human) & NA & 77 \\
\hline \multirow[t]{3}{*}{ Th1 T cell } & $\uparrow$ (mouse, human) & $\uparrow$ (mouse, human) & 58 \\
\hline & $\uparrow$ (mouse) & NA & 49 \\
\hline & $\leftrightarrow$ (human) & NA & 77 \\
\hline \multirow[t]{3}{*}{ Th17 T cell } & $\downarrow$ (mouse) & NA & NA \\
\hline & $\leftrightarrow$ (mouse) & $\leftrightarrow$ (mouse) & 58 \\
\hline & $\uparrow$ (human) & NA & 77 \\
\hline \multirow[t]{2}{*}{ Treg T cell } & $\downarrow$ (mouse, human) & $\downarrow$ (mouse, human) & 58 \\
\hline & $\downarrow$ (mouse) & NA & 49 \\
\hline B cell & $\leftrightarrow$ (human) & NA & 77 \\
\hline
\end{tabular}

Arrows indicate overall change of immune cell frequency and/or numbers in the obese intestine when compared with a lean intestine ( $\downarrow$ indicates decreased in obese state; $\uparrow$, increased in obese state; $\leftrightarrow$, no difference between obese and lean states; "mouse" and "human" indicate whether changes occur in the mouse or human intestine; "diabetes" indicates that the change is specific to a diabetic obese patient rather than a metabolically healthy obese patient; NA, data not available). Please see citations for additional details such as duration of HFD feeding.

\section{Diet-induced obesity alters the intestinal immune system}

Innate changes. Given the interplay between the microbiota, diet, and the immune system, it follows that diet-induced obesity could perhaps shape the intestinal immune system. Indeed, multiple studies have now linked HFD-related obesity to changes in intestinal PRRs, cytokines, and immune cell populations within the intestine (summarized in Figure 1 and Table 1). Using genetically engineered mouse models, changes in expression of PRRs for flagellin (TLR5), lipoproteins (TLR2), LPS (TLR4), and peptidoglycan (NOD1/2) have linked dysbiosis to the low-grade inflammatory changes of metabolic syndrome (65-67). Other PRRs, including the NLRP3 inflammasome, which is expressed by IECs, also link nutrient sensing, including saturated fatty acids, ceramide, or cholesterol crystals, to intracellular metabolic disease (68-70).

Innate immune cells express such PRRs, and changes in these cell types occur during diet-induced obesity. ILCs are important mediators of intestinal inflammation. These cells belong to the lymphoid lineage but respond to cytokines and molecular patterns in an antigen-independent manner. They are characterized by their developmental requirements and differential cytokine expression into group 1, 2, and 3 ILCs (19). The gut is enriched for group 3 ILCs (ILC3s), as these cells produce IL-17A and IL-22. During diet-induced obesity, there is a reduction in a subset of ILCs known as IL-22-producing natural cytotoxicity receptor-expressing (NCR-expressing) CD4- (NKp46+CD4-) ILC3 cells in the intestinal lamina propria of mice (58). ILC3-derived IL-22 typically supports maintenance of GALT and the anatomic barrier to commensal bacteria (71). During diet-induced obesity, IL-22 maintains mucosal integrity, and recombinant IL-22 improves metabolic parameters by targeting intestinal permeability and metabolic endotoxemia (72). Consistent with these findings, another study reported reduced levels of IL-22 as well as other cytokines known to affect intestinal barrier function, such as IL-17A, IL-17F, and IL-10, in the ileum (and colon, for IL-22) of mice after ten and 30 days of HFD feeding (49). The reduction in IL-22-producing cells in obesity may be related to a defective IL-23/IL-22-inducing axis (72).

Less is known about the role of intestinal macrophages and DCs during diet-induced obesity. However, several studies link changes in cytokines that are often expressed by antigen-presenting cells (APCs) to high-fat feeding. In the small bowel, increases in TNF- $\alpha$ have been seen after six and 16 weeks on a HFD (73), and this followed early increases in NF-кB. In the colon, the data are less consistent. Some studies have shown increased TNF- $\alpha$, but not IL-6, at the mRNA level after short- and long-duration HFD feeding $(74,75)$, while other studies have reported no differences $(73,76)$. After ten or 30 days of HFD feeding, intestinal APCs $\left(\mathrm{MHC}^{+} \mathrm{CD} 19^{-}\right)$show reduced activation markers such as CD86 and a reduced ability to induce Th17 cells in vitro, but also show an upregulation of certain inflammatory gene such as Nlrp3 (49). In humans, upper intestinal biopsies from obese subjects are associated with increased total macrophages $\left(\mathrm{CD}^{\circ} 8^{+}\right)$, mature DCs, and NK cells (77). Weight loss in humans can also affect intestinal macrophages and cytokines, as recto-sigmoid biopsies show a $25 \%$ to $75 \%$ reduction in TNF- $\alpha$, IL-1 $\beta$, IL-8, and CCL2 and decreases in macrophage and $\mathrm{T}$ cell counts with diet-induced weight loss (78). While preliminary evidence points to an increased inflammatory tone in intestinal macrophages during obesity, further studies will clarify these findings and better characterize changes in the proportions of distinct subsets of macrophages and DCs in the gut.

Other innate immune cells potentially linked to the intestine include mucosal-associated invariant $\mathrm{T}$ (MAIT) cells, eosinophils, $\gamma \delta \mathrm{T}$ cells, and neutrophils. MAIT cells, in particular, highlight another interesting connection to the gut bacteria and metabolite sensing, as they recognize bacteria through riboflavin metabolites bound to the MHC class I-related protein 1 (MR1). There is a reduction in systemic MAIT cells during obesity and type 2 diabetes in humans, with a corresponding increase in inflammatory cytokine profile inside metabolic tissue like VAT, but a detailed analysis of these cells in the intestine remains to be performed (79). Eosinophils are decreased in number and proportion within one week of HFD, and this effect correlated with increased intestinal permeability (80), but changes beyond this point require additional investigation. IL-17-producing $\gamma \delta$ T cells are increased after 12 weeks of HFD feeding in the distal small intestine and colon of mice, though a 


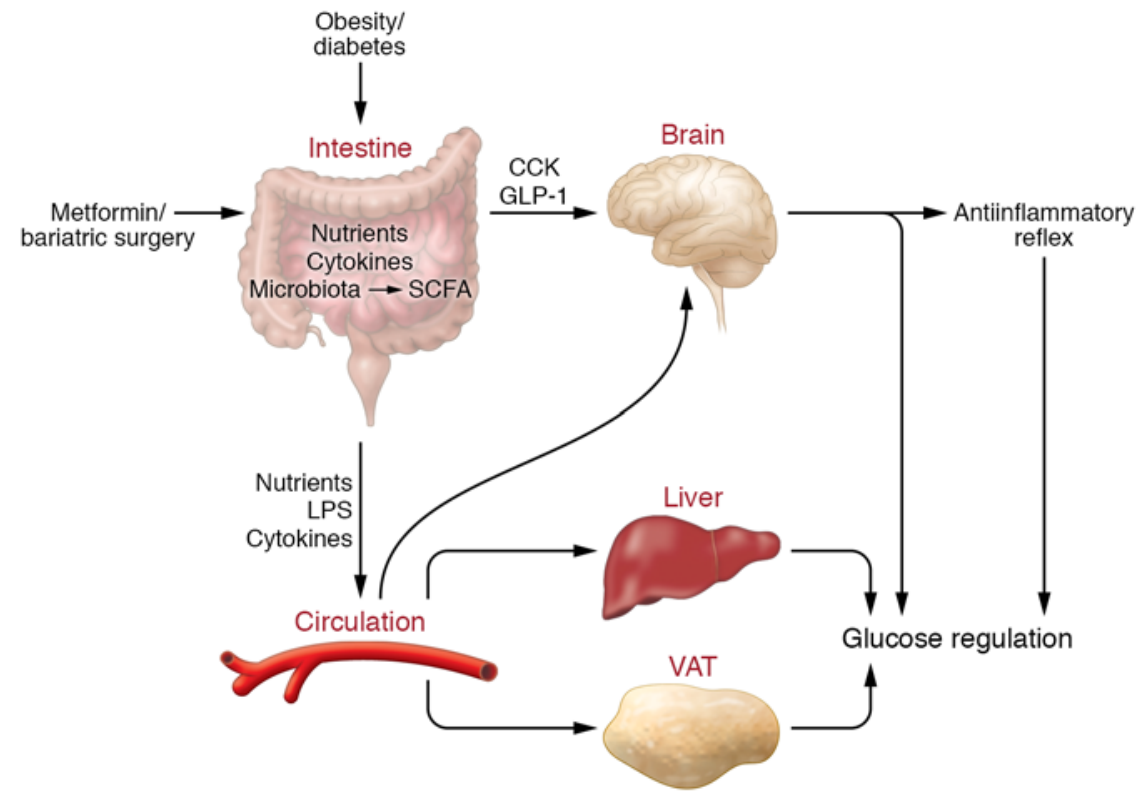

Figure 2. An integrative and neuronal-dependent intestinal immunologic and metabolic network. We propose that the intestine senses a change in the level of nutrients, cytokines, and microbiotaderived SCFAs, which triggers a gut/brain CCK- and GLP-1-dependent axis to regulate systemic inflammatory and glucose homeostasis. We further postulate that this proposed integrative and neuronal-dependent network is altered in obesity and diabetes and is targeted by metformin and bariatric surgical therapies to restore systemic metabolic homeostasis. In parallel, an influx of intestinal nutrients, bacterial products, and cytokines into the blood circulation induces inflammation and insulin resistance in the VAT and the liver and disrupts glucose homeostasis in obesity and diabetes. in the ileum, which is related to HFD-induced decreases in specific commensal bacteria such as SFB and Porphyromonas gingivalis, both of which are known to induce IL-17 (49). HFD-fed mice treated with antibiotics early on show reduced intestinal SFB, reduced ileal Th17 cells and the associated antimicrobial molecules $\beta$-defensin and RegIII $\gamma$, and increased adiposity (81). A third study identified reduced levels of IL-17 in the ileum and reduced IL-21/22 in the colons of mice on HFD (82). In contrast, one study described no change in Th17 cells at 3 or 12 weeks in mice fed a HFD (58), while a study of a comparatively large human cohort reported an increase in both Th17 and Th22 cells in the jejunums of obese subjects (77). This same study reported that there were no differences in the number of $\mathrm{CD} 2 \mathrm{O}^{+} \mathrm{B}$ cells in the lamina propria. The differences among the studies that analyzed Th17 cells likely reflect variability of microbial species, like SFB, which dictate Th17 responses (22), as well as the duration/composition of HFD and the potential for lineage plasticity within the Th17 cell population $(83,84)$. human study examining a different region of the intestine indicated no change in this population $(58,77)$. More work is also needed to determine the effect of diet on intestinal neutrophils, although one study described no histologic increases in these cells in HFD-fed mice (58).

Adaptive changes. A number of studies have characterized how obesity and/or high-fat feeding influences adaptive immune populations within the gut. While there has been some variability in this work, most studies describe an increase in IFN- $\gamma$-producing T cell populations and reductions in Foxp $3^{+}$Tregs. In the colon and distal small gut, the percentage of IFN- $\gamma$-producing Th1 cells and $\mathrm{CD} 8^{+} \mathrm{T}$ cells is increased, while Tregs are decreased after 12 weeks of HFD in mice (58). In another study the proportion of Tregs decreased and the proportion of Th1 cells increased in the ileum of mice after 30 days of HFD (49). The increase in $\mathrm{CD}^{+} \mathrm{T}$ cells, which occurs in the proximal intestine, is more prominent in the intra-epithelial fraction, and these cells are associated with a shift from CD8 $\alpha \alpha \mathrm{T}$ cells to CD8 $\alpha \beta$ T cells in humans with obesity (77). A switch from the CD8 $\alpha \alpha$ lineage, which promotes intestinal barrier integrity, may be potentially influenced by a diet lacking in vitamins, fruits, and vegetables, as these dietary constituents maintain these cells (41).

IL-17-producing $\mathrm{T}$ cells have evolved to promote mutualism with commensal bacteria to maintain the mucosal barrier and are influenced by the microbial environment (20). These cells express the AHR and ROR $\gamma$ t and secrete cytokines IL-17A, IL-17F, and IL-22, similar to intestinal ILCs. Most studies show that these cells decrease in the intestine during diet-induced obesity. Mice fed a HFD show reduced numbers of Th17 cells

\section{Intestinal regulation of systemic inflammation and glucose metabolism}

Gut immune influences. It is likely that intestinal immunologic changes incurred by diet-induced obesity represent a fundamental starting point that impinges on systemic inflammation and metabolic functioning. Mice lacking the TLR signaling molecule MyD88 in the intestine show decreased intestinal inflammation with increased Tregs and are protected from metabolic disease, suggesting that immunologic changes to gut immunity affect systemic metabolism (85). Some of these immunologic changes include increasing levels of IFN- $\gamma$, TNF- $\alpha$, and IL-1 $\beta$, coupled with reductions in IL-17 and IL-22, which would exert overall detrimental effects on intestinal barrier integrity $(49,58)$. Bacterial products and some dietary products that leak across the barrier in turn promote metabolic tissue inflammation through induction of chemokines such as CCL2, or may directly lead to lymphocyte recruitment $(40,86)$. Food antigen can also deposit in metabolic tissues such as VAT and, in conditions of poor immune tolerance such as obesity, can incite antigen-specific adaptive immunity in tissue $(58,86)$.

In addition to activation directly by bacterial or food antigen, the intestinal immune system can potentially spread its effect by dissemination of cytokines or by migration of cells. TNF- $\alpha$ or IFN- $\gamma$, which are increased in the intestines during diet-induced obesity, could potentially enter circulation and affect inflammation in distant sites. Less is known regarding whether immune cells themselves can traffic directly from the gut to metabolic tissues. Bowel immune cells, including $\mathrm{CD} 4^{+} \mathrm{T}$ cells and Tregs, possess broad trafficking abilities to lymphoid 
organs at distant sites, and while it is conceivable that they may migrate to VAT and liver, further investigation is needed (87). Interestingly, in some diets such as those with reduced amounts of vitamins A and D, intestinal immune cells upregulate systemic trafficking molecules like CCR6, which may promote migration into tissues (88).

Another means by which intestinal immunity may affect systemic metabolic control is through control of gut-derived hormones. Infusion of TNF- $\alpha$ into human subjects reduces plasma glucagon-like peptide 1 (GLP-1) (89), while chronic exposure of enteroendocrine L cells to TNF- $\alpha$ reduces GLP-1 production (90). It is uncertain whether some of these inflammatory effects on GLP-1 release are triggered by LPS or through other means, as some studies have shown a differing effect in which infusion of LPS induces GLP-1 secretion (91). Thus, it is plausible that inflammatory effects on L cell release of GLP-1 are dependent on a number of factors including the nature of the inciting agent and the chronicity of the inflammatory agents on the L cell.

Gut/brain neuronal axis and nutrient-sensing influences. Hormonal signaling in the gut may also influence systemic metabolism and inflammation through a gut/brain neuronal axis. Intestinal cholecystokinin (CCK) and GLP-1 induce a gut/brain axis to control glucose homeostasis via vagal innervation $(92,93)$. Direct stimulation of vagus nerves triggers a gut/brain/spleen axis that inhibits cytokine release from the splenic macrophages via a $\mathrm{T}$ cell-dependent cholinergic signaling axis $(94,95)$. Consistently, activation of $\mathrm{PI} 3 \mathrm{~K}$ in insulin-expressing brain neurons triggers a cholinergic, antiinflammatory reflex to limit inflammation in the fat (96), and a brain/liver axis mediates the ability of resistin to stimulate the expression of hepatic inflammatory molecules (97). Furthermore, a neuronal reflex within the gut that regulates intestinal macrophages in response to bacterial infection was recently documented (98). These findings indicate that the brain regulates whole-body metabolic and immunologic homeostasis and suggest that disruption of the gut/brain axis could lead to inflammation and glucose dysregulation in diabetes and obesity (Figure 2). In fact, nutrient sensory- and CCK-dependent mechanisms in the gut are disrupted in short-term high-fat feeding in rodents, leading to a dysregulation of hepatic glucose production $(92,99,100)$. The disruption in glucose regulation has been attributed to intestinal CCK resistance and could potentially lead to a failure to activate the cholinergic antiinflammatory pathway. It remains to be seen whether chronic low-grade intestinal inflammation, as seen in diet-induced obesity, can impinge on intestinal CCK release or resistance as it can on other intestinal hormones like GLP-1 (90). Furthermore, as gut-derived AHR ligands suppress CNS inflammation via action in astrocytes (101), it is possible that regulation of neuroinflammation by the gut directly influences food intake and energy homeostasis. Therefore, future studies investigating the relationship between dietary molecules, neuroinflammation, and metabolism are warranted.

Intestinal immune-mediated disruption of the gut barrier could potentially affect systemic metabolism through changes in gut nutrient-sensing pathways. As discussed above, HFD feeding or obesity triggers intestinal immunologic changes that promote intestinal barrier disruption. Although the time frame and mechanisms that lead to disruption of intestinal permea- bility remain unclear, increased intestinal permeability might potentially disrupt whole-body glucose homeostasis in obesity and diabetes by altering nutrient sensing in the small intestine. A key factor that regulates the initiation of nutrient-sensing mechanisms is the accumulation of preabsorptive nutrients, which is actively limited by absorption into enterocytes and entry into systemic circulation. There are several nutrient absorption mechanisms that rely primarily on the expression of specific protein transporters. For example, glucose is absorbed from the small intestine via sodium-coupled glucose transporter 1 (SGLT1) and is released from the basolateral side of enterocytes into circulation via GLUT2 (102). In contrast, fatty acids enter enterocytes via passive diffusion or through CD36 or fatty acid transport protein 4 and are secreted into circulation in the form of chylomicrons $(103,104)$. In the context of obesity, nutrients enter circulation at an increased rate, as is evident by the enhanced secretion of chylomicrons in obesity. While the mechanisms remain to be fully explored, the expression and/or activity of protein transporters that influence nutrient absorption, such as SGLT1 and CD36, are altered on a HFD and in obesity $(105,106)$. This suggests that decreased levels of preabsorptive nutrient accumulation resulting from increased apical transport may contribute to a failure in the induction of nutrient-sensing mechanisms that regulate energy homeostasis.

While in the preabsorptive state, nutrients activate various signaling events and trigger the release of gut-derived peptides such as CCK and GLP-1 to activate negative feedback, whole-body metabolic pathways that regulate food intake and glucose homeostasis $(107,108)$. In fact, direct inhibition of small intestinal CCK receptor (92) or knockdown of GLP-1 receptors in the vagal afferent nerves (93) disrupt glucose homeostasis in rodents, indicating that a gut/brain axis mediates the ability of nutrient-dependent CCK and GLP-1 action to regulate glucose homeostasis. However, the release of GLP-1 into circulation also acts on target organs in an endocrine fashion to regulate metabolic homeostasis (109).

CCK and GLP-1 are released upon nutrient accumulation and influx in enteroendocrine cells. Accumulation of esterified lipids in the small intestine is necessary for intestinal lipid sensing to trigger a CCK-dependent neuronal negative feedback pathway to lower hepatic glucose production in healthy rodents $(92,99)$. However, this intestinal lipid-dependent network is disrupted in HFD feeding, leading to a dysregulation of hepatic glucose production $(92,99)$. We propose that in the context of HFD and/or obesity, intestinal barrier leakage may prevent the ability of preabsorptive nutrients to accumulate in the small intestine, leading to an inability to trigger the gut-derived peptide network to regulate postprandial glucose homeostasis (Figure 2). In parallel, a leak of nutrients into the circulation and tissues such as liver, muscle, fat, and brain might also disrupt glucose homeostasis by inducing insulin resistance and/or inflammation in target tissues, resulting in a positive feedback loop to disrupt glucose homeostasis $(10,11,110,111)$.

As discussed above, microbiota-derived SCFAs have been implicated in maintaining intestinal immunologic tolerance. Interestingly, an acute selective rise of the SCFA propionate in the ileum activates ileal free fatty acid receptor 2 to trigger a neuronal integrative network to lower hepatic glucose production in healthy rodents (112). Although the gluco-regulatory effects of 
ileal SCFA sensing in obese and/or diabetic models remain to be investigated, the reduction of butyrate-producing bacteria seen in type 2 diabetes is prevented with anti-diabetic metformin therapy (34). These studies collectively highlight the potential converging role of intestinal SCFA sensing in the regulation of immunity and whole-body glucose homeostasis (Figure 2).

Changes in gut microbiota are not only associated with obesity and diabetes but also with changes of intestinal immunity, as well as metformin and bariatric surgical therapies (107). The potential mechanistic links between metformin and bariatric surgery as well as small intestinal nutrient-sensing mechanisms in lowering plasma glucose have been reviewed elsewhere (107). It should be noted that although metformin action in the gut can recapitulate the systemic effect of metformin in lowering plasma glucose levels in rodents (113) and people with type 2 diabetes (114), the underlying intestinal molecular events remain unclear. As changes in intestinal immunity are associated with dysbiosis, we propose that metformin therapy and bariatric surgery may potentially affect changes in gut immunity and/or microbiota to restore whole-body glucose homeostasis in obesity and diabetes. One possible link to this notion could include direct activation of AMPK in intestinal immune cells, which may promote immune cell lipid oxidation and regulatory function (115), potentially leading to reduced inflammatory potential and changes in intestinal permeability and nutrient-sensing pathways.

\section{General model and conclusions}

Overall, current data support an integrated model involving communications between the gut, liver, VAT, and brain. During chronic exposure to a Western diet and obesity, the combination of dysbiosis and increased dietary saturated fats activates TLRs and NLRs on IECs to elicit alarmins and inflammatory cytokines like IL-1 $\beta$, which can directly compromise intestinal epithelial barrier integrity (57). Dysbiosis during obesity also leads to changes in bacterial metabolite production such as reduced SCFA, while lack of dietary fruits and vegetables reduces generation of dietary AHR ligands. Together, these microbial and dietary changes are associated with immunologic changes to the intestine characterized by increased proportions of IFN- $\gamma-$ secreting Th1 and CD8 ${ }^{+}$ $\mathrm{T}$ cells and reduced proportions of Tregs, IL-22-producing ILC3 subsets, Th17 cells (in mice), and eosinophils (Table 1). The net effect of these immunologic changes is a cytokine environment that worsens intestinal barrier permeability to promote metabolic endotoxemia. Such changes to the intestinal immune system will be further influenced by genetic factors, including PRR function (116) and polymorphisms in immunologic loci (60-62), as well as the presence of other dietary factors, including food additives (117), which may alter intestinal barrier function. The ensuing leakage of bacterial and dietary products into circulation can then deposit into VAT, liver, and brain to fuel low-grade inflamma- tion and potentiate insulin resistance and glucose dysregulation. While the transport mechanisms for LPS and nutrients across the epithelial barrier are different and function independently, it is important to note that these cellular processes may also act in a synergistic manner. For instance, SGLT1 activation correlates with the opening of tight junctions, influencing small molecule/peptide influx (118). Similarly, increased chylomicron formation following long-chain fatty acid absorption increases transcellular transport of LPS (36). Importantly, while evidence suggests that altered intestinal permeability is correlated with obesity-related disease, it is currently unclear whether this is a cause and/or consequence of obesity and its metabolic complications, and thus future studies that address the relationship between intestinal permeability, inflammation, and metabolism are essential.

As described above, changes in intestinal immunity in the context of a HFD might also potentiate changes to systemic inflammation and metabolism through altered trafficking capacity of immune cells, changes to intestinal hormone secretion, direct leakage of inflammatory cytokines into circulation, and changes to nutrient-sensing pathways in the gut that communicate with the brain. In addition, changes in intestinal immunity, gut dysbiosis, and/or the production of pro-atherogenic compounds may also contribute to the development of cardiovascular disease $(119,120)$. Thus, during diet-induced obesity, the intestinal immune system acts as a central hub that potentiates systemic inflammation and ensuing metabolic consequences. Manipulation of this system may lead to new therapies for metabolic disease.

\section{Acknowledgments}

DAW laboratory is funded by the Canadian Institutes of Health Research (CIHR) (FDN-148385), the Canadian Diabetes Association (CDA) (OG-3-15-5014), the J.P. Bickell Foundation, and an Ontario Ministry of Innovation Early Researcher Award. The laboratory of TKTL is supported by a CIHR Foundation Grant (FDN143204), a CDA Operating Grant (OG-3-15-4901-TL), and the Canada Foundation for Innovation. DAW holds a Canada Research Chair in Immunometabolism. HJD is supported by postdoctoral fellowships from the CIHR and CDA. TKTL holds the John Kitson McIvor (1915-1942) Endowed Chair in Diabetes Research and the Canada Research Chair in Obesity at the Toronto General Hospital Research Institute and the University of Toronto.

Address correspondence to: Daniel Winer or Tony Lam, MaRS Centre, 101 College Street, Toronto Medical Discovery Tower, 10th floor, Toronto, Ontario, Canada M5G 1L7. Phone: 416.340.3190; Fax: 416.340.5517; E-mail: dan.winer@uhn.ca (D. Winer). Phone: 416.581.7880; Fax: 416.581.7880; E-mail: tony. lam@uhnres.utoronto.ca (T. Lam).
1. Hotamisligil GS, Shargill NS, Spiegelman BM. Adipose expression of tumor necrosis factor- $\alpha$ : direct role in obesity-linked insulin resistance. Science. 1993;259(5091):87-91.

2. Lumeng CN, Bodzin JL, Saltiel AR. Obesity induces a phenotypic switch in adipose tissue macrophage polarization. J Clin Invest.
2007;117(1):175-184.

3. Nishimura S, et al. CD8 ${ }^{+}$effector T cells contribute to macrophage recruitment and adipose tissue inflammation in obesity. Nat Med. 2009;15(8):914-920.

4. Talukdar S, et al. Neutrophils mediate insulin resistance in mice fed a high-fat diet through secreted elastase. Nat Med. 2012;18(9):1407-1412.

5. Weisberg SP, McCann D, Desai M, Rosenbaum M, Leibel RL, Ferrante AW. Obesity is associated with macrophage accumulation in adipose tissue. J Clin Invest. 2003;112(12):1796-1808.

6. Wensveen FM, et al. NK cells link obesityinduced adipose stress to inflammation 
and insulin resistance. Nat Immunol. 2015;16(4):376-385.

7. Winer DA, et al. B cells promote insulin resistance through modulation of T cells and production of pathogenic IgG antibodies. Nat Med. 2011;17(5):610-617.

8. Winer S, et al. Normalization of obesityassociated insulin resistance through immunotherapy. Nat Med. 2009;15(8):921-929.

9. $\mathrm{Xu} \mathrm{H}$, et al. Chronic inflammation in fat plays a crucial role in the development of obesity-related insulin resistance. J Clin Invest. 2003;112(12):1821-1830.

10. Lackey DE, Olefsky JM. Regulation of metabolism by the innate immune system. Nat Rev Endocrinol. 2016;12(1):15-28.

11. Tang Y, Purkayastha S, Cai D. Hypothalamic microinflammation: a common basis of metabolic syndrome and aging. Trends Neurosci. 2015;38(1):36-44.

12. McPhee JB, Schertzer JD. Immunometabolism of obesity and diabetes: microbiota link compartmentalized immunity in the gut to metabolic tissue inflammation. Clin Sci. 2015;129(12):1083-1096.

13. Chassaing B, Aitken JD, Gewirtz AT, Vijay-Kumar M. Gut microbiota drives metabolic disease in immunologically altered mice. Adv Immunol. 2012;116:93-112.

14. Winer DA, Luck H, Tsai S, Winer S. The intestinal immune system in obesity and insulin resistance. Cell Metab. 2016;23(3):413-426.

15. Abreu MT. Toll-like receptor signalling in the intestinal epithelium: how bacterial recognition shapes intestinal function. Nat Rev Immunol. 2010;10(2):131-144.

16. Johansson ME, Larsson JM, Hansson GC. The two mucus layers of colon are organized by the MUC2 mucin, whereas the outer layer is a legislator of host-microbial interactions. Proc Natl Acad Sci U S A. 2011;108(suppl 1):4659-4665.

17. Taupin DR, Kinoshita K, Podolsky DK. Intestinal trefoil factor confers colonic epithelial resistance to apoptosis. Proc Natl Acad Sci US A. 2000;97(2):799-804

18. Vaishnava $\mathrm{S}$, et al. The antibacterial lectin RegIIIgamma promotes the spatial segregation of microbiota and host in the intestine. Science. 2011;334(6053):255-258.

19. Peterson LW, Artis D. Intestinal epithelial cells: regulators of barrier function and immune homeostasis. Nat Rev Immunol. 2014;14(3):141-153.

20. Maynard CL, Elson CO, Hatton RD, Weaver CT. Reciprocal interactions of the intestinal microbiota and immune system. Nature. 2012;489(7415):231-241.

21. Round JL, Mazmanian SK. The gut microbiota shapes intestinal immune responses during health and disease. Nat Rev Immunol. 2009;9(5):313-323.

22. Ivanov II, et al. Induction of intestinal Th17 cells by segmented filamentous bacteria. Cell. 2009;139(3):485-498.

23. Wu HJ, et al. Gut-residing segmented filamentous bacteria drive autoimmune arthritis via $\mathrm{T}$ helper 17 cells. Immunity. 2010;32(6):815-827.

24. Round JL, et al. The Toll-like receptor 2 pathway establishes colonization by a commensal of the human microbiota. Science.
2011;332(6032):974-977.

25. Atarashi K, et al. Induction of colonic regulatory $\mathrm{T}$ cells by indigenous Clostridium species. Science. 2011;331(6015):337-341.

26. Furusawa Y, et al. Commensal microbederived butyrate induces the differentiation of colonic regulatory T cells. Nature. 2013;504(7480):446-450.

27. Lukovac S, et al. Differential modulation by Akkermansia muciniphila and Faecalibacterium prausnitzii of host peripheral lipid metabolism and histone acetylation in mouse gut organoids. MBio. 2014;5(4):e01438-14.

28. Bäckhed F, et al. The gut microbiota as an environmental factor that regulates fat storage. Proc Natl Acad Sci U S A. 2004;101(44):15718-15723.

29. Turnbaugh PJ, Ley RE, Mahowald MA, Magrini V, Mardis ER, Gordon JI. An obesity-associated gut microbiome with increased capacity for energy harvest. Nature. 2006;444(7122):1027-1031.

30. Ridaura VK, et al. Gut microbiota from twins discordant for obesity modulate metabolism in mice. Science. 2013;341(6150):1241214.

31. Karlsson FH, et al. Gut metagenome in European women with normal, impaired and diabetic glucose control. Nature. 2013;498(7452):99-103.

32. Le Chatelier E, et al. Richness of human gut microbiome correlates with metabolic markers. Nature. 2013;500(7464):541-546.

33. Qin J, et al. A metagenome-wide association study of gut microbiota in type 2 diabetes. Nature. 2012;490(7418):55-60.

34. Forslund $\mathrm{K}$, et al. Disentangling type 2 diabetes and metformin treatment signatures in the human gut microbiota. Nature. 2015;528(7581):262-266.

35. Cani PD, et al. Changes in gut microbiota control metabolic endotoxemia-induced inflammation in high-fat diet-induced obesity and diabetes in mice. Diabetes. 2008;57(6):1470-1481.

36. Ghoshal S, Witta J, Zhong J, de Villiers W, Eckhardt E. Chylomicrons promote intestinal absorption of lipopolysaccharides. JLipid Res. 2009;50(1):90-97.

37. Birchenough GM, Nyström EE, Johansson ME, Hansson GC. A sentinel goblet cell guards the colonic crypt by triggering Nlrp6-dependent Muc2 secretion. Science. 2016;352(6293):1535-1542.

38. Amar J, et al. Intestinal mucosal adherence and translocation of commensal bacteria at the early onset of type 2 diabetes: molecular mechanisms and probiotic treatment. EMBO Mol Med. 2011;3(9):559-572.

39. Cani PD, et al. Metabolic endotoxemia initiates obesity and insulin resistance. Diabetes. 2007;56(7):1761-1772.

40. Caesar R, Tremaroli V, Kovatcheva-Datchary P, Cani PD, Bäckhed F. Crosstalk between gut microbiota and dietary lipids aggravates WAT inflammation through TLR signaling. Cell Metab. 2015;22(4):658-668.

41. Kiss EA, et al. Natural aryl hydrocarbon receptor ligands control organogenesis of intestinal lymphoid follicles. Science. 2011;334(6062):1561-1565

42. Lee JS, et al. AHR drives the development of gut ILC22 cells and postnatal lymphoid tissues via pathways dependent on and independent of
Notch. Nat Immunol. 2011;13(2):144-151.

43. Veldhoen M, Brucklacher-Waldert V. Dietary influences on intestinal immunity. Nat Rev Immunol. 2012;12(10):696-708.

44. Shi H, Kokoeva MV, Inouye K, Tzameli I, Yin H, Flier JS. TLR4 links innate immunity and fatty acid-induced insulin resistance. JClin Invest. 2006;116(11):3015-3025.

45. Oh DY, et al. A Gpr120-selective agonist improves insulin resistance and chronic inflammation in obese mice. Nat Med. 2014;20(8):942-947.

46. Cui G, et al. Liver X receptor (LXR) mediates negative regulation of mouse and human Th17 differentiation. J Clin Invest. 2011;121(2):658-670.

47. $\mathrm{Hu} \mathrm{X}$, et al. Sterol metabolism controls $\mathrm{T}(\mathrm{H}) 17$ differentiation by generating endogenous ROR $\gamma$ agonists. Nat Chem Biol. 2015;11(2):141-147.

48. Fang S, et al. Intestinal FXR agonism promotes adipose tissue browning and reduces obesity and insulin resistance. Nat Med. 2015;21(2):159-165.

49. Garidou L, et al. The gut microbiota regulates intestinal CD4 $\mathrm{T}$ cells expressing ROR $\gamma \mathrm{t}$ and controls metabolic disease. Cell Metab. 2015;22(1):100-112.

50. Sun CM, et al. Small intestine lamina propria dendritic cells promote de novo generation of Foxp3 T reg cells via retinoic acid. J Exp Med. 2007;204(8):1775-1785.

51. Bruce D, Cantorna MT. Intrinsic requirement for the vitamin D receptor in the development of CD $8 \alpha \alpha$-expressing T cells. JImmunol. 2011;186(5):2819-2825.

52. Rooks MG, Garrett WS. Gut microbiota, metabolites and host immunity. Nat Rev Immunol. 2016;16(6):341-352.

53. Thorburn AN, Macia L, Mackay CR. Diet, metabolites, and "western-lifestyle" inflammatory diseases. Immunity. 2014;40(6):833-842.

54. Koh A, De Vadder F, Kovatcheva-Datchary P, Bäckhed F. From dietary fiber to host physiology: short-chain fatty acids as key bacterial metabolites. Cell. 2016;165(6):1332-1345.

55. Zelante T, et al. Tryptophan catabolites from microbiota engage aryl hydrocarbon receptor and balance mucosal reactivity via interleukin-22. Immunity. 2013;39(2):372-385.

56 . Veldhoen $\mathrm{M}$, et al. The aryl hydrocarbon receptor links TH17-cell-mediated autoimmunity to environmental toxins. Nature. 2008;453(7191):106-109.

57. Al-Sadi RM, Ma TY. IL-1beta causes an increase in intestinal epithelial tight junction permeability. J Immunol. 2007;178(7):4641-4649.

58. Luck H, et al. Regulation of obesity-related insulin resistance with gut anti-inflammatory agents. Cell Metab. 2015;21(4):527-542.

59. Hasnain SZ, et al. IL-10 promotes production of intestinal mucus by suppressing protein misfolding and endoplasmic reticulum stress in goblet cells. Gastroenterology. 2013;144(2):357-368.e9.

60. Belforte FS, Coluccio Leskow F, Poskus E, Penas Steinhardt A. Toll-like receptor $4 \mathrm{D} 299 \mathrm{G}$ polymorphism in metabolic disorders: a meta-analysis. Mol Biol Rep. 2013;40(4):3015-3020.

61. Hua Y, Shen J, Song Y, Xing Y, Ye X. Interleu- 
kin-10 -592C/A, -819C/T and -1082A/G polymorphisms with risk of type 2 diabetes mellitus: a HuGE review and meta-analysis. PLoS One. 2013;8(6):e66568.

62. Saxena M, Srivastava N, Banerjee M. Association of IL-6, TNF- $\alpha$ and IL-10 gene polymorphisms with type 2 diabetes mellitus. Mol Biol Rep. 2013;40(11):6271-6279.

63. Revelo XS, Winer S, Winer DA. Starving Intestinal Inflammation with the amino acid sensor GCN2. Cell Metab. 2016;23(5):763-765.

64. Gulhane M, et al. High fat diets induce colonic epithelial cell stress and inflammation that is reversed by IL-22. Sci Rep. 2016;6:28990.

65. Denou E, et al. Defective NOD2 peptidoglycan sensing promotes diet-induced inflammation, dysbiosis, and insulin resistance. EMBO Mol Med. 2015;7(3):259-274.

66. Vijay-Kumar M, et al. Metabolic syndrome and altered gut microbiota in mice lacking Toll-like receptor 5. Science. 2010;328(5975):228-231.

67. Caricilli AM, et al. Gut microbiota is a key modulator of insulin resistance in TLR 2 knockout mice. PLoS Biol. 2011;9(12):e1001212.

68. Vandanmagsar B, et al. The NLRP3 inflammasome instigates obesity-induced inflammation and insulin resistance. Nat Med. 2011;17(2):179-188.

69. Wen H, et al. Fatty acid-induced NLRP3-ASC inflammasome activation interferes with insulin signaling. Nat Immunol. 2011;12(5):408-415.

70. Yan Y, et al. Omega-3 fatty acids prevent inflammation and metabolic disorder through inhibition of NLRP3 inflammasome activation. Immunity. 2013;38(6):1154-1163.

71. Sonnenberg GF, et al. Innate lymphoid cells promote anatomical containment of lymphoid-resident commensal bacteria. Science. 2012;336(6086):1321-1325.

72. Wang $X$, et al. Interleukin-22 alleviates metabolic disorders and restores mucosal immunity in diabetes. Nature. 2014;514(7521):237-241.

73. Ding S, et al. High-fat diet: bacteria interactions promote intestinal inflammation which precedes and correlates with obesity and insulin resistance in mouse. PLoS One. 2010;5(8):e12191.

74. Lam YY, et al. Increased gut permeability and microbiota change associate with mesenteric fat inflammation and metabolic dysfunction in diet-induced obese mice. PLoS One. 2012;7(3):e34233.

75. Liu Z, et al. Diet-induced obesity elevates colonic TNF- $\alpha$ in mice and is accompanied by an activation of Wnt signaling: a mechanism for obesity-associated colorectal cancer. J Nutr Biochem. 2012;23(10):1207-1213.

76. Li H, et al. Intestinal, adipose, and liver inflammation in diet-induced obese mice. Metab Clin Exp. 2008;57(12):1704-1710.

77. Monteiro-Sepulveda M, et al. Jejunal T cell inflammation in human obesity correlates with decreased enterocyte insulin signaling. Cell Metab. 2015;22(1):113-124.

78. Pendyala S, Neff LM, Suárez-Fariñas M, Holt PR. Diet-induced weight loss reduces colorectal inflammation: implications for colorectal carcinogenesis. Am J Clin Nutr. 2011;93(2):234-242.

79. Magalhaes I, et al. Mucosal-associated invariant $\mathrm{T}$ cell alterations in obese and type 2 diabetic patients. JClin Invest. 2015;125(4):1752-1762.

80. Johnson AM, et al. High fat diet causes depletion of intestinal eosinophils associated with intestinal permeability. PLoS One. 2015;10(4):e0122195.

81. Cox LM, et al. Altering the intestinal microbiota during a critical developmental window has lasting metabolic consequences. Cell. 2014;158(4):705-721.

82. Cavallari JF, Denou E, Foley KP, Khan WI, Schertzer JD. Different Th17 immunity in gut, liver, and adipose tissues during obesity: the role of diet, genetics, and microbes. Gut Microbes. 2016;7(1):82-89.

83. Gagliani N, et al. Th17 cells transdifferentiate into regulatory $\mathrm{T}$ cells during resolution of inflammation. Nature. 2015;523(7559):221-225.

84. Hirota K, et al. Fate mapping of IL-17-producing $\mathrm{T}$ cells in inflammatory responses. Nat Immunol. 2011;12(3):255-263.

85. Everard A, et al. Intestinal epithelial MyD88 is a sensor switching host metabolism towards obesity according to nutritional status. Nat Commun. 2014;5:5648.

86. Wang Y, et al. T-lymphocyte responses to intestinally absorbed antigens can contribute to adipose tissue inflammation and glucose intolerance during high fat feeding. PLoS One. 2010;5(11):e13951.

87. Morton AM, Sefik E, Upadhyay R, Weissleder R, Benoist C, Mathis D. Endoscopic photoconversion reveals unexpectedly broad leukocyte trafficking to and from the gut. Proc Natl Acad Sci U S A. 2014;111(18):6696-6701.

88. Chang JH, Cha HR, Lee DS, Seo KY, Kweon MN. 1,25-Dihydroxyvitamin D3 inhibits the differentiation and migration of $\mathrm{T}(\mathrm{H}) 17$ cells to protect against experimental autoimmune encephalomyelitis. PLoS One. 2010;5(9):e12925.

89. Lehrskov-Schmidt L, Lehrskov-Schmidt L, Nielsen ST, Holst JJ, Møller K, Solomon TP. The effects of TNF- $\alpha$ on GLP-1-stimulated plasma glucose kinetics. JClin Endocrinol Metab. 2015;100(4):E616-E622.

90. Gagnon J, et al. Chronic exposure to TNF $\alpha$ impairs secretion of glucagon-like peptide-1. Endocrinology. 2015;156(11):3950-3960.

91. Nguyen AT, et al. Lipopolysaccharides-mediated increase in glucose-stimulated insulin secretion: involvement of the GLP-1 pathway. Diabetes. 2014;63(2):471-482.

92. Cheung GW, Kokorovic A, Lam CK, Chari M, Lam TK. Intestinal cholecystokinin controls glucose production through a neuronal network. Cell Metab. 2009;10(2):99-109.

93. Krieger JP, Arnold M, Pettersen KG, Lossel P, Langhans W, Lee SJ. Knockdown of GLP-1 receptors in vagal afferents affects normal food intake and glycemia. Diabetes. 2016;65(1):34-43.

94. Tracey KJ. Physiology and immunology of the cholinergic antiinflammatory pathway. JClin Invest. 2007;117(2):289-296.

95. Rosas-Ballina M, et al. Acetylcholinesynthesizing $\mathrm{T}$ cells relay neural signals in a vagus nerve circuit. Science. 2011;334(6052):98-101.

96. Wang L, et al. Pten deletion in RIP-Cre neurons protects against type 2 diabetes by activating the anti-inflammatory reflex. Nat Med.
2014;20(5):484-492.

97. Muse ED, Lam TK, Scherer PE, Rossetti L. Hypothalamic resistin induces hepatic insulin resistance. J Clin Invest. 2007;117(6):1670-1678.

98. Gabanyi I, Muller PA, Feighery L, Oliveira TY, Costa-Pinto FA, Mucida D. Neuro-immune interactions drive tissue programming in intestinal macrophages. Cell. 2016;164(3):378-391.

99. Wang PY, et al. Upper intestinal lipids trigger a gut-brain-liver axis to regulate glucose production. Nature. 2008;452(7190):1012-1016.

100.Rasmussen BA, et al. Duodenal activation of cAMP-dependent protein kinase induces vagal afferent firing and lowers glucose production in rats. Gastroenterology. 2012;142(4):834-843.e3.

101. Rothhammer V, et al. Type I interferons and microbial metabolites of tryptophan modulate astrocyte activity and central nervous system inflammation via the aryl hydrocarbon receptor. Nat Med.2016;22(6):586-597.

102. Wright EM, Loo DD, Hirayama BA. Biology of human sodium glucose transporters. Physiol Rev. 2011;91(2):733-794.

103. Nassir F, Wilson B, Han X, Gross RW, Abumrad NA. CD36 is important for fatty acid and cholesterol uptake by the proximal but not distal intestine. J Biol Chem. 2007;282(27):19493-19501.

104.Stahl A, et al. Identification of the major intestinal fatty acid transport protein. Mol Cell. 1999;4(3):299-308.

105. Nguyen NQ, et al. Accelerated intestinal glucose absorption in morbidly obese humans: relationship to glucose transporters, incretin hormones, and glycemia. JClin Endocrinol Metab . 2015;100(3):968-976.

106. Buttet M, et al. Deregulated lipid sensing by intestinal CD36 in diet-induced hyperinsulinemic obese mouse model. PLoS One. 2016;11(1):e0145626.

107. Duca FA, Bauer PV, Hamr SC, Lam TK. Glucoregulatory relevance of small intestinal nutrient sensing in physiology, bariatric surgery, and pharmacology. Cell Metab. 2015;22(3):367-380.

108. Cote CD, Zadeh-Tahmasebi M, Rasmussen BA, Duca FA, Lam TK. Hormonal signaling in the gut. J Biol Chem. 2014;289(17):11642-11649.

109.Drucker DJ. Deciphering metabolic messages from the gut drives therapeutic innovation: the 2014 Banting Lecture. Diabetes. 2015;64(2):317-326.

110.Samuel VT, Shulman GI. The pathogenesis of insulin resistance: integrating signaling pathways and substrate flux. JClin Invest. 2016;126(1):12-22.

111. Reilly SM, Saltiel AR. Obesity: a complex role for adipose tissue macrophages. Nat Rev Endocrinol. 2014;10(4):193-194.

112. Zadeh-Tahmasebi M, et al. Activation of short and long chain fatty acid sensing machinery in the ileum lowers glucose production in vivo. J Biol Chem. 2016;291(16):8816-8824.

113. Duca FA, et al. Metformin activates a duodenal Ampk-dependent pathway to lower hepatic glucose production in rats. Nat Med. 2015;21(5):506-511.

114. Buse JB, et al. The primary glucose-lowering effect of metformin resides in the gut, not the circulation: results from short-term pharmacokinetic and 12-week dose-ranging studies. Diabetes 
Care. 2016;39(2):198-205.

115. Michalek RD, et al. Cutting edge: distinct glycolytic and lipid oxidative metabolic programs are essential for effector and regulatory $\mathrm{CD} 4^{+} \mathrm{T}$ cell subsets. J Immunol. 2011;186(6):3299-3303.

116. Wlodarska M, et al. NLRP6 inflammasome orchestrates the colonic host-microbial interface by regulating goblet cell mucus secretion. Cell.
2014;156(5):1045-1059.

117. Chassaing B, et al. Dietary emulsifiers impact the mouse gut microbiota promoting colitis and metabolic syndrome. Nature. 2015;519(7541):92-96.

118. Turner JR, et al. Physiological regulation of epithelial tight junctions is associated with myosin light-chain phosphorylation. Am J Physiol.
1997;273(4 pt 1):C1378-C1385.

119. Yamashita $\mathrm{T}$, et al. Intestinal immunity and gut microbiota as therapeutic targets for preventing atherosclerotic cardiovascular diseases. Circ J. 2015;79(9):1882-1890.

120. Wang Z, et al. Gut flora metabolism of phosphatidylcholine promotes cardiovascular disease. Nature. 2011;472(7341):57-63. 